\title{
АЛГОРИТМ МОРФОЛОГИЧЕСКОЙ ОБРАБОТКИ РАДИОЛОКАЦИОННЫХ ИЗОБРАЖЕНИЙ И АВТОМАТИЧЕСКОГО ОБНАРУЖЕНИЯ ОБЪЕКТОВ ПО РАДИОЛОКАЦИОННОЙ ТЕНИ
}

\author{
В. П. Лихачев, М. А. Пантюхин, С. В. Сидоренко
}

ВУНЦ ВВС «Военно-воздушная академия им. проф. Н. Е. Жуковского и Ю. А. Гагарина» (г. Воронеж)

\author{
Поступила в редакцию 16.04.2018 г.
}

\begin{abstract}
Аннотация. В статье обосновывается последовательность операций обработки радиолокационных изображений, обеспечивающих автоматическое обнаружение и селекцию объектов по их радиолокационной тени. В отличие от существующих алгоритмов с постоянным уровнем ложной тревоги (CFAR - Constant False Alarm Rate) предложено применять морфологические операции над инвертированным бинарным изображением. Определены параметры морфологических операций, которые обеспечивают устранение искажений радиолокационной тени объектов и исключение из «области интереса» (ROI - Regions of Interes) объектов, радиолокационные тени которых отличаются от эталонных (рассчитанных исходя из габаритов объекта и условий его съемки). Алгоритм работает при полном отсутствии сведений о параметрах плотности распределения вероятности отсчетов отметки объекта и фона. Приведены результаты обработки радиолокационных изображений реальных объектов, расположенных на естественном фоне.

Ключевые слова: радиолокационная тень объектов, морфологические операции, устранение искажений, пространственная корреляция, автоматическое обнаружение.

Annotation. The article substantiates the sequence of operations for processing radar images, which provide automatic detection and selection of objects by their radar shadow. Unlike existing detection algorithms with a constant level of false alarm (CFAR-Constant False Alarm Rate), it is suggested to apply morphological operations on an inverted binary image. The parameters of morphological operations that ensure the elimination of distortions of the radar shadow of the object and the exclusion from the "area of interest" (ROI-Regions of Interest) of objects whose radar shade differs from the reference ones (calculated based on the size of the object and the conditions for its recording) are determined. The algorithm works in the complete absence of information about the parameters of the probability distribution density of the sample counts of the object and the background. The results of processing radar images of real objects located on a natural background are given.
\end{abstract}

Keywords: radar shadow of the object, morphological operations, elimination of distortions, spatial correlation, automatic detection.

\section{ВВЕДЕНИЕ}

Радиолокационные станции с цифровым синтезированием апертуры антенны (РСA) являются одним из основных источников информации при решении разных прикладных задач [1-5]. Автоматическое обнаружение радиолокационных ориентиров и объектов с заданными габаритами при определении 2018

() Лихачев В. П., Пантюхин М. А., Сидоренко С. В., области интереса $[6,7]$ может быть осуществлено путем определения локальных статистических характеристик изображений с последующим сравнением рассчитанных характеристик с задаваемыми пороговыми значениями [8-10]. В ряде случаев отметки объектов на радиолокационных изображений (РЛИ) имеют интенсивность, соизмеримую с интенсивностью фона, а их радиолокационная тень (РЛТ), как правило, имеет большой контраст по сравнению с фоном. Если отметки объектов на РЛИ могут быть сымитиро- 


\section{В. П. Лихачев, М. А. Пантюхин, С. В. Сидоренко}

ваны $[11,12]$, то имитация их РЛТ считается практически неразрешимой [2].

При малой высоте полета носителя РСА или большой высоте объектов (радиолокационных ориентиров) их РЛТ является одним из главных демаскирующих признаков, т. к. размеры РЛТ на изображении значительно превышают размеры отметки объекта.

Часто считается, что РЛТ антропогенных объектов однородны и на РЛИ наблюдаются на фоне спекл-шума [7]. Однако область РЛТ объекта может быть искажена внутренними шумами приемника, частичной когерентностью радиоголограммы [13], а также высокими объектами естественного происхождения на земной поверхности (бугры, растения и т. п.), что может привести к появлению неоднородности РЛТ и потере информации о габаритах объектов или к возникновению ложных (искаженных) РЛТ. Указанные обстоятельства обуславливают необходимость предварительной обработки РЛИ, обеспечивающей устранение подобных искажений РЛТ объектов.

\section{АНАЛИЗ РЕЗУЛЬТАТОВ ПРЕДШЕСТВУЮЩИХ РАБОТ}

Существует два типичных способа выделения на РЛИ областей интереса, используемых затем для автоматического распознавания находящихся там объектов: сегментация изображения и оценка информативных признаков. При сегментировании изображений некоторые методы [14-18] для распознавания цели используют только отметку цели, и при этом пренебрегают информацией о габаритах цели, которая также содержится в ее РЛТ. Из-за нечеткого контура отметок целей на РЛИ возможны ошибки сегментации и потеря полезной для распознавания информации $[7,14]$. Использование РЛТ цели на РЛИ с высоким разрешением приводит к инвариантности способов автоматизированного распознавания цели от их радиолокационной отражательной способности и существенно повышает эффективность распознавания (снижается вероятность ложной тревоги) [19, $20,23]$.
На серии последовательных РЛИ обнаруживают истинное местоположение быстро и медленно движущихся целей по смещению их РЛТ [21, 22].

Предложены способы улучшения РЛТ обычных объектов [24], высоких и узких объектов [25], и объектов с плоскими горизонтальными структурами [26] на основе компенсации фазовых искажений радиоголограммы и коррекции параметров реальной РЛТ по параметрам РЛТ, вычисленной с учетом высоты носителя РСА.

Искажения радиолокационной тени объектов на РЛИ предложено устранять использованием ее геометрической проекции с географической привязкой на цифровую карту местности [27]. Различие характеристик РЛТ истинных и ложных целей предложено использовать в нейронном алгоритме распознавания [28].

Алгоритмы сегментации элементов изображений [29] предполагают фильтрацию потенциальных областей интереса. Сегментация РЛТ [19-28] повышает вероятность обнаружения целей, но эффективность алгоритмов распознавания цели по ее РЛТ ограничена из-за увеличения ложных тревог [30]. Для устранения негативных эффектов на аэрофотоснимках в оттенках серого [33, 37] или на цветных [34], на инфракрасных [35] и радиолокационных изображениях зачастую используется аппарат математической морфологии [31, 32].

Для создания используемых в операциях математической морфологии структурных элементов, используется генетический алгоритм, который обеспечивает инвариантность алгоритма CFAR к размеру и форме обнаруженной морфологической совокупности пикселей [36]

В статьях [38-40] предлагаются способы автоматического обнаружения изменений на паре РЛИ высокого разрешения, полученных в различное время PCA TerraSAR-X (Германия). Усовершенствованные морфологические операции (на основе вычисления морфологических профилей разностных характеристик) не требуют определения структурных элементов, а для описания специфи- 
ческих участков амплитудных РЛИ высокого разрешения используются профили атрибутов [40]: коэффициент вариации и нормированную эффективную площадь рассеяния пикселей. В процедуре сравнения РЛИ одних и тех же областей используется эвклидово расстояние между векторами-профилями атрибутов первого и следующих сегментированных амплитудных РЛИ $[38,40]$.

В ряде работ $[41,42]$ предлагается способ автоматического распознавания наземной цели на РЛИ после его разделения на три части: целевую область, тень и фон, которые обрабатывают раздельно. Причем сама РЛТ используется только для выделения области интереса без изменения отметки цели на изображении. Исходное изображение и изображение «цель + фон» классифицируются по отдельным эталонным изображениям, а результаты классификации объединяются путем суммирования уровня баллов для распознавания цели [41]. Для выявления несоответствия между различными типами целей в [43] применяют морфологическую операцию открытия над остатками разности интенсивностей пикселей отметки цели на РЛИ и пикселей ее радиолокационного эталона.

Если задача восстановления краёв теневой области объектов на РЛИ с высоким разрешением, искажаемых траекторными нестабильностями носителя РСА во время записи радиоголограммы, естественным фоном или другими факторами, достаточно успешно решена ранее [35-43], то алгоритмы устранения искажений РЛТ из-за попадания в ее внутреннюю область других объектов практически не рассматривались.

Вместе с тем остается мало исследованным, какая последовательность морфологических операций [31] (дилатация (расширения), эрозия (уменьшения) и производных от них - замыкание (closing), размыкание (opening) и др.) является целесообразной при обработке РЛИ, обеспечивающей повышение качества обнаружения объектов в условиях искажения внутренней области РЛТ.

В связи с указанным целью работы является обоснование последовательности процедур обработки РЛИ с использованием аппарата математической морфологии в интересах повышения качества автоматического обнаружения объектов по их РЛТ.

\section{МАТЕРИАЛЫ И МЕТОДЫ ИССЛЕДОВАНИЯ}

Первая процедура обработки - бинаризация РЛИ $\left(S=\left\{S_{x y}\right\}\right.$, где $x=1 \ldots X, X$ - максимальное значение строк в РЛИ, $y=1 \ldots Y, Y$ максимальное значение столбцов в РЛИ), обусловлена тем, что операции математической морфологии наиболее эффективны при обработке бинарных изображений.

Бинаризация РЛИ по порогу предполагает перевод изображения в градациях серого в монохромное, где присутствуют только два типа пикселей (темные и светлые).

Выбор порога как значения уровня яркости, где $L$ - максимальное значение яркости $t=0, \ldots, L$, для бинаризации РЛИ предлагается производить методом Отсу $[44,45]$, который предполагает разделение на два класса всех объектов на изображении (фон и объект). Строится нормализованная гистограмма изображения по значениям:

$$
p_{i}=\frac{n_{i}}{N},
$$

где $N=X Y$ - общее количество пикселей на изображении, $n_{i}$ - количество пикселей с уровнем яркости $i$.

Для каждого класса вычисляются параметры:

$$
\begin{gathered}
\omega_{0}(t)=\sum_{i=1}^{t} p_{i}, \omega_{1}(t)=1-\omega_{0}(t), \\
\mu_{0}(t)=\frac{\sum_{i=1}^{t} i p_{i}}{\omega_{0}(t)}, \mu_{1}(t)=\frac{\sum_{i=t+1}^{L} i p_{i}}{\omega_{1}(t)} \\
\sigma_{b}^{2}(t)=\omega_{0}(t) \omega_{1}(t)\left(\mu_{0}(t)-\mu_{1}(t)\right)^{2}
\end{gathered}
$$

где $\omega_{0}(t), \omega_{1}(t)$ - относительные частоты двух классов 0 и 1 , разделенных порогом $t ; \mu_{0}(t)$, $\mu_{1}(t)$ - средние уровни для каждого из двух классов 0 и 1 изображения, разделенных порогом $t ; \sigma_{b}^{2}(t)$ - межклассовая дисперсия.

Искомое значение порога $t$ соответствует максимальному значению $\sigma_{b}^{2}(t)$.

Следующая процедура обработки РЛИ инвертирование пикселей изображения $n_{i}$ 
обусловлена тем, что морфологические операции не предназначены для работы с объектами на изображении типа РЛТ и т. п., так как на бинарном радиолокационном изображении они относятся к фону. После инвертирования исходного изображения границы РЛТ объекта могут иметь искажения, что обусловливает следующий этап - исследование влияния различных морфологических операций на РЛИ.

Для разделения объектов на РЛИ применялась одна из базовых морфологических операций - «эрозия». Изображение преобразуется в множество $A$. «Эрозия» множества $A$ по множеству $B$ - это множество всех таких точек $Z$, при сдвиге в которые множество $B$ целиком содержится в множестве $A$ [31]. Эрозию $A$ по $B$ формально записывается в виде:

$$
C=\left\{Z \mid(B)_{z} \subseteq A\right\} .
$$

В выражении (5) переменная $B$ - это структурообразующее множество (примитив), например, симметричная единичная матрица размером $N_{p} \times N_{p}$, в которой начальной точкой является центральный пиксель.

Так как за один проход примитивом удаляется контур в $N_{p}-1$ пикселей вокруг объекта, то в результате применения данной операции РЛТ объекта может быть удалена. Поэтому установим, что допустимое количество итераций операции $C$ определяется как:

$$
M \leq \operatorname{int}\left\{\frac{\min \left\{l_{m 1}, l_{m 2}\right\}}{\max \{\Delta x, \Delta y\}}\right\}-\left(N_{p}-1\right),
$$

где $M$ - число повторений операции «эрозия», int $\{x\}$ - целая часть $x, \min \{x, y\}$ - минимальное значение $x, y, \max \{x, y\}$ - максимальное значение $x, \quad y, \Delta x=\frac{c}{2 \Delta f_{c} \cos \alpha}$, $\Delta y=\frac{\lambda h}{2 V T_{c} \sin \alpha}-$ разрешающая способность на РЛИ по наклонной и путевой дальности, $l_{m 1}=\frac{h_{1}}{\operatorname{tg}(\alpha)}, \quad l_{m 2}=l_{1}|\cos (\phi)|+l_{2}|\sin (\phi)|$ и $h_{1}-$ длина, ширина и высота РЛТ объекта, $c$ скорость света, $\Delta f_{c}$ - ширина спектра зондирующего сигнала, $l_{1}, l_{2}$ - длина и ширина объекта соответственно.
Негативным эффектом, возникающим после применения операции «эрозия», является появление разрывов в области РЛТ объекта. Этот эффект возникает в случае, когда рядом с обнаруживаемым объектом находятся объекты выше него. Разрывы внутри РЛТ объекта можно определить как область фона, окруженную связным бордюром из элементов переднего плана. Заполнение таких областей предложено проводить на основе операций дилатации, дополнения и пересечения множеств [31]. Для этого формируется массив $X_{0}$ с размерами равными размеру исходного РЛИ, содержащий на множестве $A$ нули во всех точках, кроме мест разрывов, которым присваивается значение 1. Заполнение всех разрывов производится с использованием следующей рекуррентной процедуры:

$$
X_{k}=\left(X_{k-1} \oplus B\right) \cap A^{c},
$$

где $k=1,2,3 \ldots K$ - число разрывов на изображении, $\oplus$ - знак строгой дизъюнкции, $\cap-$ знак пересечения множеств, $A^{c}-$ множество $A$ после применения операции (5).

Процедура (7) останавливается на $k$-м шаге, когда $X_{k}=X_{k-1}$, а последующее объединение множеств $X_{k}$ и $A^{c}$ приводит к устранению разрывов.

Автоматическое обнаружение объекта на РЛИ предполагает применение адаптивной процедуры формирования эталона РЛТ $(U)$ объекта, в ходе которой производится расчет его параметров $\Delta x, \Delta y, l_{m 1}, l_{m 2}$ и $h_{1}$.

Перед процедурой обнаружения объекта предлагается сформировать множество объектов за счет объединения пикселей с учетом 8-ми связной структурой соседства [31], а также вычислить площади образуемой фигуры.

После этого производится фильтрация РЛИ, т. е. площадь эталона последовательно сравнивается с площадью каждого образованного объекта из сформированного множества, и если их площадь значительно больше или меньше теоретически рассчитанной, то они удаляются.

Для конечного принятия решения об обнаружении объекта с конкретными характеристиками предлагается использовать проце- 
дуру пространственной корреляции $(K r)$ эталона РЛТ (может быть рассчитан по $l_{m 1}$ и $l_{m 2}$, исходя из условий съемки и линейных размеров объекта или взят из заранее подготовленной базы данных эталонов) и сформированного после вышеуказанных процедур изображения.

Пространственная корреляция представляет собой процесс движения маски фильтра по изображению и вычисления суммы произведений значений элементов маски и значений пикселей, на которые попадают соответствующие элементы маски, для всех точек изображения [31].

Таким образом, предложенный алгоритм имеет структуру, представленную на рис. 1.

\section{РЕЗУЛЬТАТЫ ИССЛЕДОВАНИЙ И ИХ ОБСУЖДЕНИЕ}

Результаты бинаризации исходного РЛИ здания с РЛТ (рис. 2,а высота полета носителя PCA $h=270$ м, скорость полета $V=27$ м/с, время синтезирования апертуры антенны $T_{c}=1,5$ с, угол визирования $\alpha=30^{\circ}$, угол между продольной осью объекта и направлением на носитель РСА в азимутальной плоскости составляет $\phi=90^{\circ}$ ) по порогу, определенному в соответствии с выражениями (1)-(4), представлены на рис. 2,6. Радиоголограмма получена с помощью РСА, установленной на беспилотный летательный аппарат «Мерлин-21Б» (АО «НИИ СТТ», г. Смоленск).
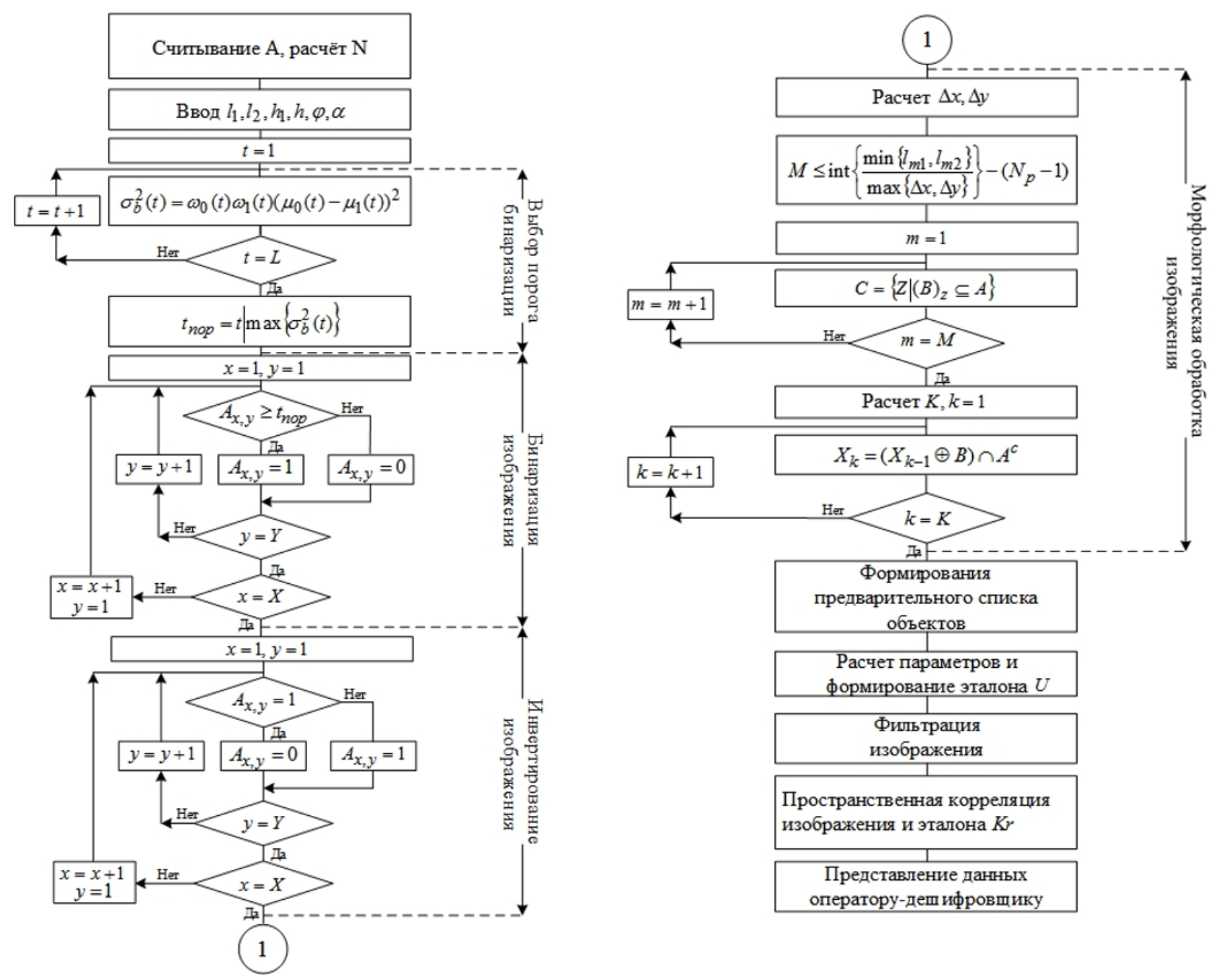

Рис. 1. Структурная схема алгоритма автоматического обнаружения РЛТ объектов с использованием морфологической обработки 


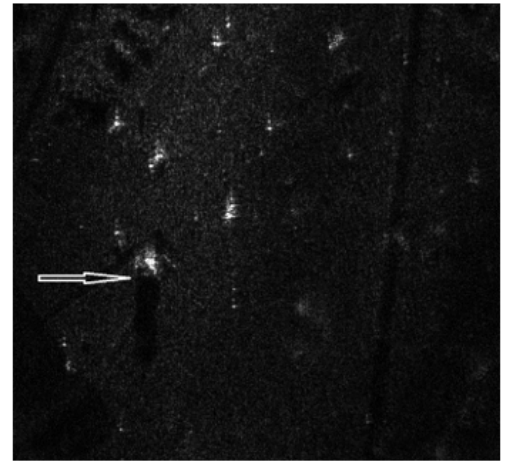

a)

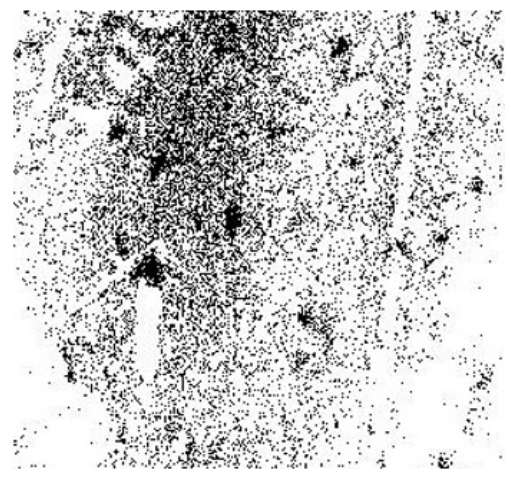

в)

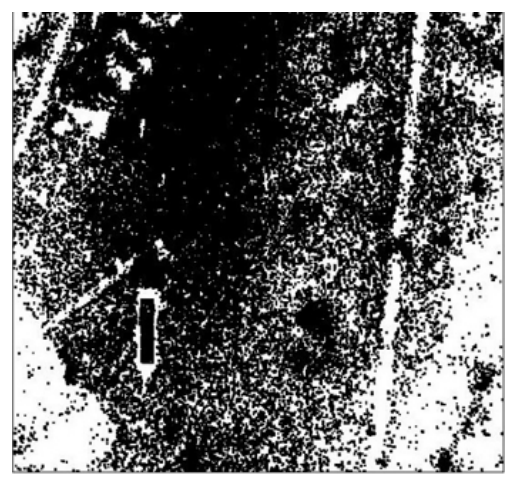

d)

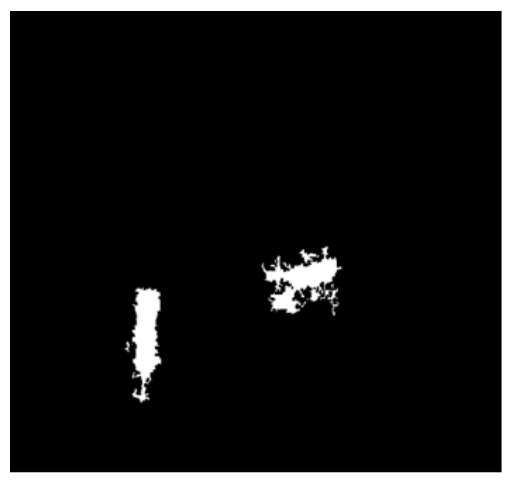

H)

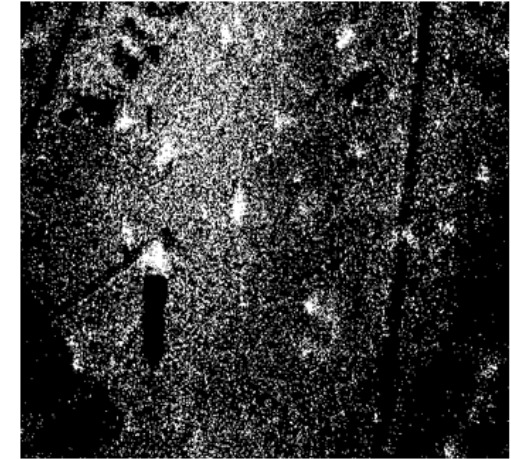

6)

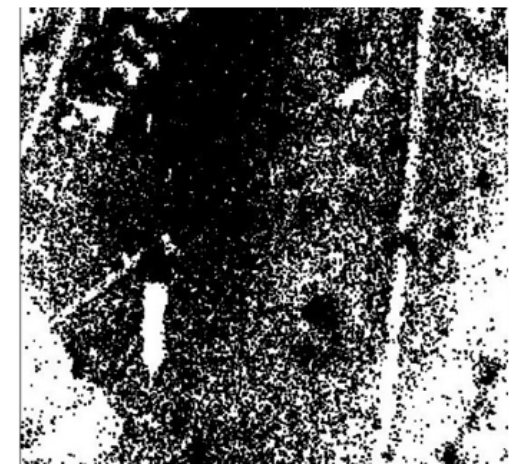

2)

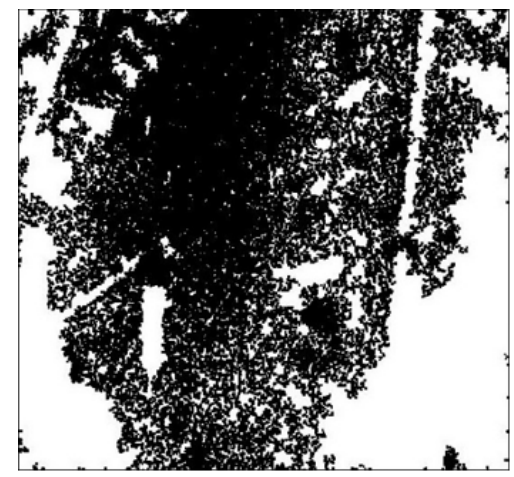

e)

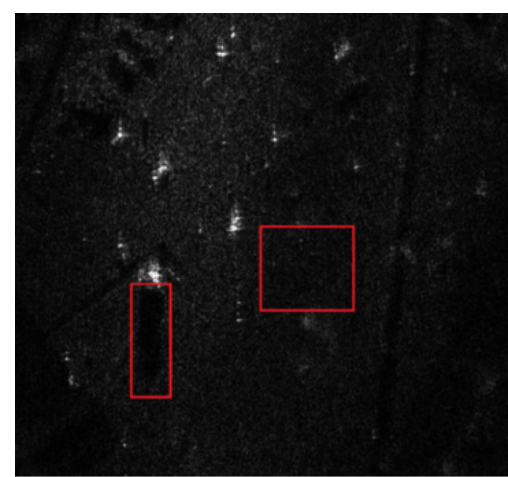

3)

Рис. 2. Исходное РЛИ объекта и его обработка предложенным алгоритмом 


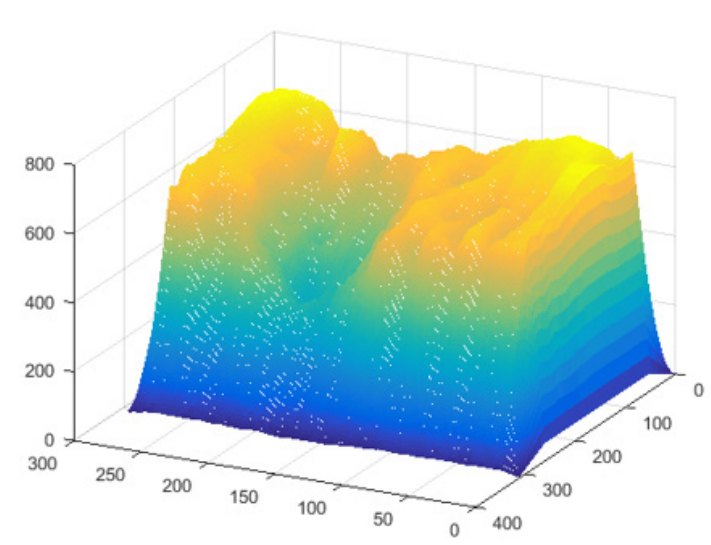

а) исходного изображения и эталона

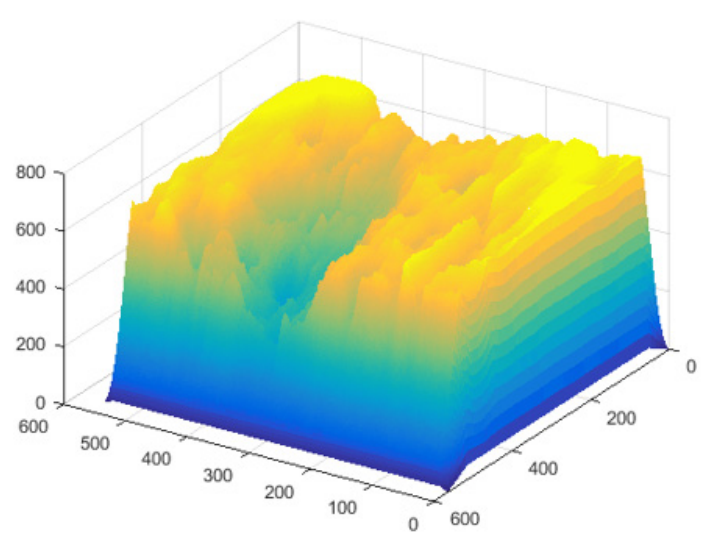

б) искаженного изображения и эталона

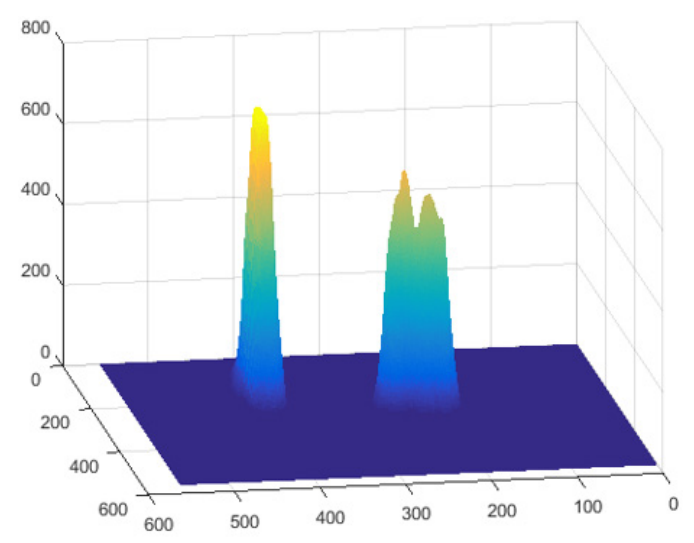

в) обработанного изображения и эталона

Рис. 3. Результаты вычисления пространственной корреляиии РЛИ и эталона РЛТ здания

Результаты выполнения операции инвертированиия пикселей РЛИ, представленного на рис. 2,б, показаны на рис. 2,в.

Далее исследовалось влияния различных морфологических операций на устранение искажений РЛТ здания. Эмпирическим путем выявлена следующая последовательность применения морфологических операций.

Согласно (6) для объекта (рис. 2 a), имеющего габариты: $l_{1}=12 \mathrm{~m}, l_{2}=10 \mathrm{M}, h_{1}=6 \mathrm{~m}$, при значениях $\Delta x=0,375 \mathrm{~m}, \Delta y=0,2 \mathrm{~m}$, рассчитано $M=8$. Откуда следует, что при локации протяженных объектов с субметровым разрешением $M$ будет достаточно большим, и для выделения РЛТ объекта операцию «эрозия» целесообразно ограничить 5-6 повторениями. На рис. 2,г представлены результаты выполнения процедуры (5) над бинарным инвертированным РЛИ.

Для проверки эффективности операций дилатации, дополнения и пересечения множеств (7) в исходном РЛИ примерно 70 \% об- ласти РЛТ здания искусственно было заменено на область фона, яркость пикселей которых выше выбранного порога, при этом контур РЛТ оставался не измененным (рис. 2,д). На рис. 2,е показан результат последовательного применения вышеизложенных морфологических операций. Результат фильтрации сформированного списка объектов, представлен на рис. 2,ж. Объекты, площади РЛТ которых сопоставимы с теоретически рассчитанной площадью РЛТ, выделяются на исходном изображении (рис. 2,3). Как видно из рис. 2,ж на изображении, во-первых, отфильтровываются отметки объектов с такими же габаритами, но с меньшей РЛТ, а, во-вторых, помимо необходимых объектов выделяются ложные отметки с РЛТ, параметры которых близки к параметрам эталонной РЛТ.

На рис. 3 представлены результаты вычисления значений корреляционной функции $K r$ исходного а), смоделированного б), обработанного в) изображений и эталона. 


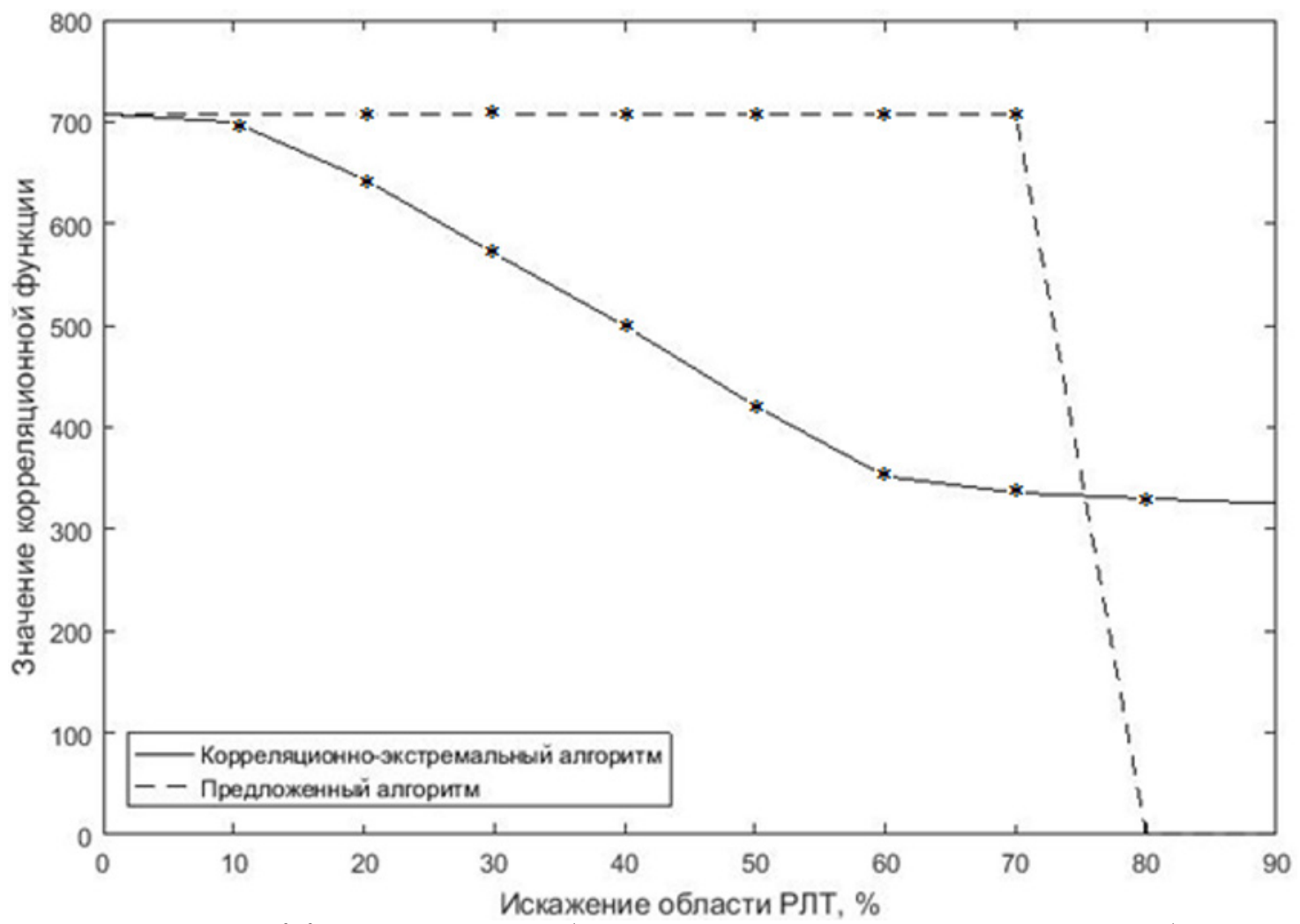

Рис. 4. Сравнение эфбективности работы алгоритмов автоматического обнаружения в условиях искажения области РЛТ объекта

Из анализа корреляционных функций, представленных на рис. 3, видно, что только на рис. 3,в имеется глобальный максимум, по которому возможно с большой достоверностью обнаружить заданный объект, определить его координаты и расположение (ориентацию) на местности.

Эффективность предложенного алгоритма для условий искажения от 0 до 90 \% РЛТ объекта оценили путем сравнения результатов его работы с результатами корреляционно-экстремального алгоритма. На рис. 4 представлены зависимости корреляционной функции $\mathrm{Kr}$ от искажения области РЛТ.

Анализ зависимостей, представленных на рис. 3, а,6 показал, что при вычислении пространственной корреляции исходного РЛИ имеется очень много (около 175000 пикселей) ложных обнаружений РЛТ объекта, в связи с этим определить наличие объекта и его местоположение не представляется возможным. А после обработки РЛИ предложенным алгоритмом отфильтровываются объекты, размеры РЛТ которых не соответствуют размерам РЛТ искомого объекта, в связи с чем корреляционная функция имеет один четко выраженный максимум (рис. 3,6).

Из анализа зависимостей, показанных на рис. 4, следует: при наличии искажений от $0 \%$ до $10 \%$ от площади РЛТ объекта предлагаемый и корреляционно-экстремальный алгоритм имеют одинаковую эффективность; предлагаемый алгоритм инвариантен к искажениям в пределах от $10 \%$ до 70 \% от площади РЛТ объекта, а затем происходит резкое снижение его эффективности до нуля (изображение искаженной РЛТ уничтожается); эффективность корреляционно-экстремального алгоритма снижается линейно от $100 \%$ до 50 \% при увеличении искажений РЛТ объекта от $10 \%$ до $60 \%$ от его площади.

Для определения помехоустойчивости предложенного алгоритма (рис. 1) проведено имитационное моделирование: к РЛИ добавлялся шум с нулевым математическим ожиданием и дисперсией $\sigma_{u}^{2}$. Установлено, что алгоритм сохраняет работоспособность при соотношении $\frac{\sigma_{\phi}^{2}}{\sigma_{u}^{2}}=-53$ дБ, где $\sigma_{\phi}^{2}-$ дисперсия фона на изображении. На рис. 5 представ- 


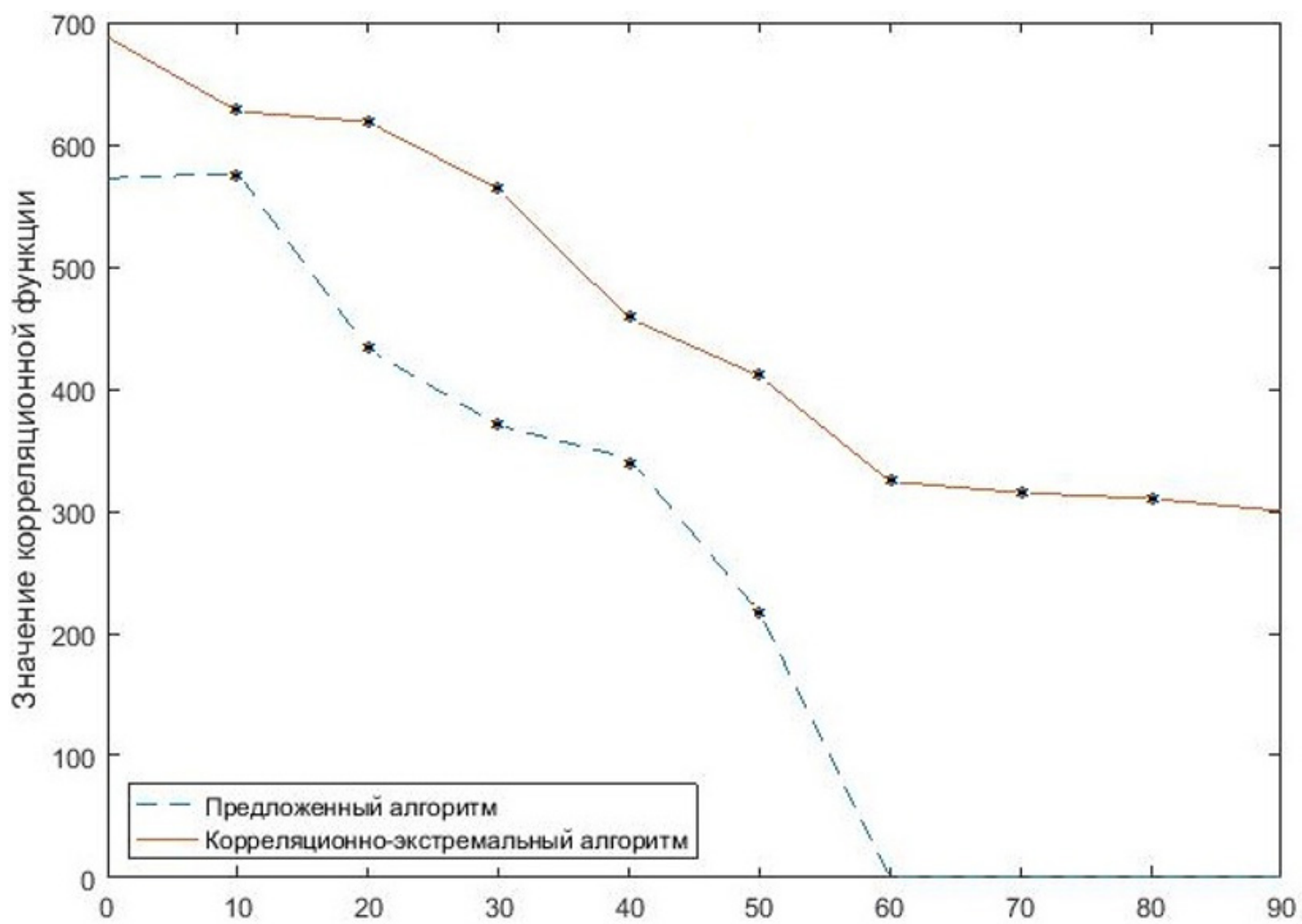

Рис. 5. Сравнение эффективности работы алгоритмов автоматического обнаружения в условиях искажения области РЛТ объекта, при $\frac{\sigma_{\phi}^{2}}{\sigma_{u}^{2}}=-53$ дБ

лены зависимости корреляционной функции от искажения области РЛТ при $\frac{\sigma_{\phi}^{2}}{\sigma_{w}^{2}}=-53$ дБ.

Из графиков на рис. 5 видно, что, в данных условиях, значение корреляционной функции $K r$, вычисленной после обработки РЛИ предложенным алгоритмом меньше чем при корреляционно-экстремальном алгоритме. Однако при обнаружении объектов корреляционно-экстремальным алгоритмом четко выраженный экстремум функции не соответствует отметке объекта и при различных реализациях шума его координаты на РЛИ изменяются случайным образом (как правило соответствуют затененной области фона). При искажении РЛТ объекта до 50 \% предложенный алгоритм однозначно определяет местоположение искомого объекта. При искажении РЛТ объекта больше чем на 50 \% при $\frac{\sigma_{\phi}^{2}}{\sigma_{u}^{2}}=-53$ дБ происходит «размытие» границы РЛТ шумом и, как следствие, существенное уменьшение ее размеров, в результате чего она удаляется на этапе фильтрации.

\section{ЗАКЛЮЧЕНИЕ}

Разработанная последовательность операций с использованием морфологической обработки радиолокационных изображений позволяет автоматически выделять на них интересующие объекты (даже если амплитуда сигналов, отраженных локальными центрами рассеивания самого объекта, соизмерима с амплитудой сигналов, отраженных фоном) по радиолокационной тени, в том числе, с искаженной внутренней областью. Кроме этого, предложенный алгоритм обеспечивает исключение из «области интереса» тех объектов, радиолокационные тени которых отличаются от эталонных (т. е. рассчитанных исходя из габаритов объекта и условий его съемки). Однако, при искажении более чем 70 \% площади РЛТ объекта использовать данный алгоритм невозможно, т. к. происходит пропуск объекта на РЛИ. Полученные результаты обработки РЛИ, сформированного РСА, позволяют рекомендовать предложенный алгоритм для: автономной навигации пилоти- 
руемых и беспилотных летательных аппаратов; автоматического обнаружения объектов на РЛИ; автоматизации процесса дешифрирования РЛИ, который, по мнению широкого круга специалистов, является наиболее трудоемким и требующим большого промежутка времени процессом.

\section{СПИСОК ЛИТЕРАТУРЫ}

1. Авиационные системы радиовидения: монография / под ред. Г. С. Кондратенкова. Москва : Радиотехника, 2015. - 648 с.

2. Купряшкин, И. Ф. Космическая радиолокационная съемка земной поверхности в условиях помех: монография / И. Ф. Купряшкин, В. П. Лихачев. - Воронеж : Издательско-полиграфический центр «Научная книга», 2014. - 460 c.

3. Болкунов, А. А. Оценка радиолокационной заметности вооружения, военной и специальной техники с применением беспилотных летательных аппаратов / А. А. Болкунов, Л. Б. Рязанцев, С. В. Сидоренко // Военная мысль. - 2017. - № 9. - С. 70.

4. Лихачев, В. П. Применение беспилотных летательных аппаратов для ведения тактической радиолокационной разведки / В. П. Лихачев, Л. Б. Рязанцев, И. Ю. Чередников // Военная мысль. - 2016. - № 3. - С. 24.

5. Способ формирования радиолокационного изображения : пат. 2578126 Российская Федерация : МПК G 01 S 13/00 / Лихачев В. П., Рязанцев Л. Б., Трущинский А. В., Купряшкин И. Ф. ; заявитель и патентообладатель Воронеж. Федеральное государственное казенное военное образовательное учреждение высшего профессионального образования «Военный учебно-научный центр Военно-воздушных сил «Военно-воздушная академия им. проф. Н. Е. Жуковского и Ю. А. Гагарина». - № 2014149401/07 ; заявл. 08.12.14 ; опубл. 20.03.16, Бюл. № 8. - 9 с.

6. Устройство формирования радиолокационного изображения в радиолокационной станции с синтезированной апертурой антенны : пат. 2619771 Российская Федерация : МПК G 01 S 13/90 / Купряшкин И. Ф., Лихачев В. П., Рязанцев Л. Б., Яковенков В. В. ; за- явитель и патентообладатель Смоленск. ЗАО «Научно-исследовательский институт современных телекоммуникационных технологий». - № 2016104062 ; заявл. 08.02.16 ; опубл. 18.05.17, Бюл. № 14. - 11 с.

7. Automatic Target Recognition in Synthetic Aperture Radar Imagery / El-Darymli, K. [and others] // A State-of-the-Art Review. IEEE Access. - 2016. - № 4. - Р. 6014-6058.

8. Системный подход к решению проблем автоматического дешифрирования радиолокационных изображений наземных объектов в реальном масштабе времени / В. А. Кузнецов [и др.] // Вестник Воронеж. гос. ун-та. Сер. Системный анализ и информационные технологии. - 2017. - № 2. - С. 5-15.

9. Сuрота, А. А. Двухэтапный алгоритм обнаружения и оценивания границы объектов на изображении в условиях аддитивных помех и деформирующих искажений / А. А. Сирота, А. И. Соломатин, Е. В. Воронова // Компьютерная оптика. - 2010. - Т. 34, № 1. - C. 109-117.

10. Соломатин, А. И. Обнаружение и оценивание границ объектов на изображениях в условиях аддитивного шума и деформирующих искажений [Электронный ресурс] / А. И. Соломатин // Воронеж: ВГУ. 2011. Режим доступа: http://www.dissercat.com/ content/obnaruzhenie-i-otsenivanie-granitsobektov-na-izobrazheniyakh-v-usloviyakhadditivnogo-shuma\#ixzz5AmbEoqHC. - (Дата обращения 20.03.2018).

11. Способ искажения радиолокационного изображения в космической радиолокационной станции с синтезированной апертурой антенны : пат. 2622904 Российская Федерация : МПК G 01 S 13/90, G 01 S 7/38 / Купряшкин И. Ф., Лихачев В. П., Селезнев Д. А., Усов Н. А.; заявитель и патентообладатель Смоленск. 3АО «Научно-исследовательский институт современных телекоммуникационных технологий». - № 2016113470 ; заявл. 07.04.16; опубл. 21.06.17, Бюл. № 18. - 19 с.

12. Лихачев, В. П. Показатель помехоустойчивости РЛС с синтезированной апертурой антенны к параметрическим формируемым помехам, имитирующим точечные объекты / 
В. П. Лихачев, В. В. Семенов, А. А. Веселков // Антенны. - 2017. - № 12 (244). - С. 31.

13. Лихачев, В. П. Формирование радиолокационных изображений летательных аппаратов методом обращенного синтезирования апертуры в условиях частичной когерентности сигнала / В. П. Лихачев, А. Я. Пасмуров // Радиотехника и электроника. - 1999. - Т. 44. № 3. - С. 294.

14. Park, J. New discrimination features for SAR automatic target recognition / J. Park, S. Park, K. Kim // IEEE Geosci. Remote Sens. Lett. - 2013. - № 10. - P. 476.

15. Neighborhood geometric center scaling embedding for SAR ATR / Y. L. Huang [and others] // IEEE Trans. Aerosp. Electron. Syst. 2014. - № 50. - P. 180-192.

16. Target recognition in synthetic aperture radar images using binary morphological operations / B. Y. Ding [and others] // J. Appl. Remote Sens. - 2016. - № 10. - P. 46.

17. Amoon, M. Automatic target recognition of synthetic aperture radar (SAR) images based on optimal selection of Zernike moment features / M. Amoon; G. Rezai-rad // IET Comput. Vis. - 2014. № 8. - P. 77.

18. Anagnostopulos, G. C. SVM-based target recognition from synthetic aperture radar images using target region outline descriptors / Anagnostopulos G. C. // Nonlinear Anal. - 2009. № 71. - P. 2934-2939.

19. Papson, S. Classification via the shadow region in SAR imagery / S. Papson, R. M. Narayanan // IEEE Trans. Aerosp. Electron. Syst. - 2012. - № 48. - P. 969-980.

20. Cui, J. J. Automatic recognition of MSTAR targets using radar shadow and super resolution features // J. J. Cui, J. Gudnason, M. Brookes // In Proceedings of the IEEE International Conference on Acoustics, Speech, and Signal Processing (ICASSP). 2005. - P. 589-592.

20. Cui, J. Radar shadow and super resolution features for automatic recognition of MSTAR targets / J. Cui, J. Gudnason, M. Brookes // IEEE International Radar Conference - 2005. - P. 534539.

21. Jahangir, M. Detecting Moving Targets in SAR Imagery Through Shadow Tracking / M. Ja- hangir // In Proceedings of EUSAR. - 2006. P. $1-4$.

22. $X u, H$. Shadow-aided method for ground slow moving targets detection of airborne high-resolution SAR images / H. Xu [and others] // 2015 IEEE 5th Asia-Pacific Conference on Synthetic Aperture Radar (APSAR), Singapore. 2015. - P. 831-834.

23. Donnell, B. P. Using Shadows To Detect Targets in Synthetic Aperture Radar Imagery / B. P. Donnell // Air Force Institute of Technology Graduate School of Engineering and Management (AFIT/EN), Degree of Master of Science in Electrical Engineering. - 2009. - 96 p.

24. A Novel Approach for Shadow Enhancement in High-Resolution SAR Images Using the Height-Variant Phase Compensation Algorithm / Y. Zhang [and others] // in IEEE Geoscience and Remote Sensing Letters. - vol. 10 № 1. - 2013. P. 189-193.

25. An approach for shadow enhancement about tall and narrow targets in SAR images / Y. Zhang [and others] // 2015 IEEE International Geoscience and Remote Sensing Symposium (IGARSS), Milan. - 2015. - P. 3263-3265.

26. The shadow enhancement for targets with flat structures in SAR images / Y. Zhang [and others] // 2016 IEEE International Geoscience and Remote Sensing Symposium (IGARSS), Beijing. - 2016. - P. 921-924.

27. Prasath, V. B. S. Radar shadow detection in synthetic aperture radar images using digital elevation model and projections [Электронный pecypc] / V. B. S. Prasath, O. Haddad // Journal of Applied Remote Sensing, 8(1), 083628. - 2014. P. 7. - Режим доступа: https://www.researchgate. net/publication/256465152 Pre-print available at arXiv:1309.1830. - (Дата обращения 5.03.18).

28. SAR deception jamming target recognition based on the shadow feature / X. Tang [and others] // 25th European Signal Processing Conference (EUSIPCO), Kos. - 2017. - P. 2491-2495.

29. Жердев, Д. А. Распознавание объектов на радиолокационных изображениях с использованием показателей сопряженности и опорных подпространств / Д. А. Жердев, Л.Н.Казанский, В. А. Фурсов // Компьютерная оптика. - 2015. - Т. 39, № 2. - С. 255. 
30. Schumacher, R. Non-cooperative target identification of battlefield targets - Classification results based on SAR images / R. Schumacher, J. Schiller // In Proceedings of the IEEE International Radar Conference, Arlington, VA, USA. 2005. - P. 167-172.

31. Гонсалес, Р. Цифровая обработка изображений / Р. Гонсалес, Р. Вудс - Москва : Издво Техносфера, 2012. - 1104 с.

32. Огнев, И. В. Обработка изображений методами математической морфологии в ассоциативной осцилляторной среде / И. В. Огнев, Н. А. Сидорова // Известия высших учебных заведений. Поволжский регион. Технические науки. - 2007. - № 4. - С. 87-97.

33. Tsai, V. J. D. Automatic shadow detection and radiometric restoration on digital aerial images/ V. J. D. Tsai // in Proc. IGARSS, Toulouse, France, Jul. 21-25. - 2003. - P. 732-733.

34. Tsai, V. J. D. Automated Shadow Compensation in Color Aerial Images [Электронный peсурс] / V. J. D. Tsai, J. Victor // Paper presented at the ASPRS 2006 Annual Conference, Reno, Nevada. - 2006. - Режим доступа: https://pdfs. semanticscholar.org/3dc5/201797f7a2082d699f 8684d867d2c59d0660.pdf. - (Дата обращения 24.03.2018).

35. Farajzadeh, M. Detection of Small Target Based on Morphological Filters / M. Farajzadeh, A. Mahmoodi, M. R. Arvan // Electrical Engineering (ICEE), 20th Iranian Conference, Tehran, Iran - 2012. - P. 1097 - 1101.

36. Bhanu, B. Genetic algorithm based feature selection for target detection in SAR images / B. Bhanu, Y. Lin // Image and Vision Computing. - Volume 21, Issue 7. -2003. - P. 591-608.

37. Beant, K. Mathematical morphological edge detection for remote sensing images / B. Kaur, A. Garg // Proc. 3rd International Conference on Electronics Computer Technology. 2011. - P. 324-327.

Лихачев Владимир Павлович - д-р техн. наук, профессор, профессор кафедры ВУНЦ ВBC «Военно-воздушная академия им. проф. Н. Е. Жуковского и Ю. А. Гагарина».

E-mail: lvp_home@mail.ru
38. Using morphological differential attribute profiles for change ategorization in high resolution SAR images / M. Boldt [and others] // International Archives of the Photogrammetry, Remote Sensing and Spatial Information Sciences. - Volume XL-1/W1. - ISPRS Hannover Workshop, Germany. - 2013. - P. 29-34.

39. Yamada, Y. Relation between ground features and mathematical morphology using JERS1/SAR data during flooding time in paddy areas / Yamada Y. // Proceedings. 2003 IEEE International Geoscience and Remote Sensing Symposium (IGARSS '03). - 2003. - P. 2517.

40. SAR Image Segmentation Using Morphological Attribute Profiles / M. Boldt [and others] // The International Archives of the Photogrammetry, Remote Sensing and Spatial Information Sciences, Volume XL-3, 2014. ISPRS Technical Commission III Symposium, 5 - 7 September 2014, Zurich, Switzerland. - P. 39-44.

41. Chang, M. Target Recognition in SAR Images Based on Information-Decoupled Representation / M. Chang, X. You // Remote Sens. 2018. - 10(1). - P. 138.

42. Wang, G. A novel target detection method for SAR images based on shadow proposal and saliency analysis / G. Wang, J. You, H. Zhou // Neurocomputing. - 2017. - V. 267.- P. 220-231.

43. Target recognition in synthetic aperture radar images using binary morphological operations / B. Y. Ding [and others] //Appl. Remote Sens. - 2016. - 10. - 046006.

44. Otsu, N. A. Threshold selection method from gray - level histograms / N. A. Otsu // IEEE Trans. Sys., Man., Cyber. - 1979. - P. 62-66.

45. Liao, P. A. Fast Algorithm for Multilevel Thresholding / P. Liao, T. Chen, P. Chung // J. Inf. Sci. Eng. - 2017. - P. 713-727.

Likhachev Vladimir P. - D.Sc in Engineering, professor, professor Air Force Education and Research Center «The Zhukovsky and Gagarin Air Force Academy».

E-mail: lvp_home@mail.ru 
Пантюхин Максим Александрович - канд. техн. наук, начальник научно-исследовательской лаборатории ВУНЦ ВВС «Военно-воздушная академия им. проф. Н. Е. Жуковского и Ю. А. Гагарина».

E-mail: ol-max@mail.ru

Сидоренко Сергей Викторович - адъюнкт ВУНЦ ВВС «Военно-воздушная академия им. проф. Н. Е. Жуковского и Ю. А. Гагарина». E-mail: sidor-vire@rambler.ru
Pantyukhin Maksim A. - Head of Research Laboratory Air Force Education and Research Center «The Zhukovsky and Gagarin Air Force Academy».

E-mail: ol-max@mail.ru

Sidorenko Sergey V. - post-graduate student Air Force Education and Research Center «The Zhukovsky and Gagarin Air Force Academy». E-mail: sidor-vire@rambler.ru 